\title{
The Role of Modular Robotics in Mediating Nonverbal Social Exchanges
}

\author{
Marti, P; Giusti, L; Lund, Henrik Hautop
}

Published in:

I E E E Transactions on Robotics

Link to article, DOI:

10.1109/TRO.2009.2020346

Publication date:

2009

Document Version

Publisher's PDF, also known as Version of record

Link back to DTU Orbit

Citation (APA):

Marti, P., Giusti, L., \& Lund, H. H. (2009). The Role of Modular Robotics in Mediating Nonverbal Social

Exchanges. I E E E Transactions on Robotics, 25(3), 602-613. https://doi.org/10.1109/TRO.2009.2020346

\section{General rights}

Copyright and moral rights for the publications made accessible in the public portal are retained by the authors and/or other copyright owners and it is a condition of accessing publications that users recognise and abide by the legal requirements associated with these rights.

- Users may download and print one copy of any publication from the public portal for the purpose of private study or research.

- You may not further distribute the material or use it for any profit-making activity or commercial gain

- You may freely distribute the URL identifying the publication in the public portal

If you believe that this document breaches copyright please contact us providing details, and we will remove access to the work immediately and investigate your claim. 


\title{
The Role of Modular Robotics in Mediating Nonverbal Social Exchanges
}

\author{
Patrizia Marti, Leonardo Giusti, and Henrik Hautop Lund
}

\begin{abstract}
This paper outlines the use of modular robotics to encourage and facilitate nonverbal communication during therapeutic intervention in dementia care. A set of new socially interactive modular robotic devices called rolling pins (RPs) has been designed and developed to assist the therapist in interacting with dementiaaffected patients. The RPs are semitransparent plastic tubes that are capable of measuring their orientation and the speed of their rotation; at a local level, they have three types of feedback: red, green, and blue light, sound, and vibration. The peculiarity of the RPs is that they are able to communicate with each other or with other devices equipped with the same radio communication technology. The RPs are usually used in pairs, as the local feedback of an RP can be set depending not only on its own speed and orientation but also on the speed and the orientation of the peer RP. The system is not used as a therapeutic tool per se but as a facilitator and a mediator of social dynamics during normal therapy to counteract social isolation that can result in dementia through the loss of social skills. An experiment is reported that shows that by using the RPs, the patients participated in the activity by coordinating their behavior with the therapist and imitating the same interaction patterns generated by the therapist.
\end{abstract}

Index Terms-Dementia, gesture-based interaction, imitation, modular robotics, social exchanges, tangible media.

\section{INTRODUCTION}

$\mathbf{T}$ HIS PAPER describes an early experiment using modular robotic devices to mediate social exchanges between the therapist and dementia-affected patients with the objective to facilitate the development of ordinary therapeutic intervention. One of the problems of current therapeutic practice is the difficulty for the therapist to establish and maintain a sufficient level of communication with the patient during therapy. Dementia-affected subjects suffer from an acquired permanent neurodegenerative disorder that affects the global functioning of the individual progressively impairing cognition, personality, and behavior. In particular, dementia is strongly characterized by social isolation and difficulties in communication. Speech becomes increasingly inefficient, and progressive shortterm memory difficulties and problems with new learning make conversations and other social interactions increasingly problematic [1]. The social sphere of the individual is jeopardized

Manuscript received September 22, 2008; revised February 14, 2009 and March 22, 2009. First published May 5, 2009; current version published June 5, 2009. This paper was recommended for publication by Associate Editor E. Guglielmelli and Editor K. Lynch upon evaluation of the reviewers' comments.

P. Marti is with the University of Siena, 53100 Siena, Italy (e-mail: marti@unisi.it).

L. Giusti is with the University of Siena, 53100 Siena, Italy, and also with the Fondazione Bruno Kessler, 38050 Povo, Italy (e-mail: giusti@ media.unisi.it).

H. H. Lund is with the Technical University of Denmark, 2800 Kongens Lyngby, Denmark (e-mail: hhl@playware.dtu.dk).

Digital Object Identifier 10.1109/TRO.2009.2020346 not only by the impairment of social abilities resulting from the global functional impairment of the subject but also by the voluntary withdrawal from relational exchanges due to a number of contextual factors ranging from aural and visual ability impairment, institutionalization, interpersonal disorientation, and lack of self-esteem and motivation.

Furthermore, in a longitudinal study that lasted 12 years and involved 2812 noninstitutionalized elderly persons, Bassuk et al. [2] showed how social disengagement is a risk factor for cognitive impairment among elderly persons. These results have a direct implication on the definition of care interventions: If elderly persons are actively provided with opportunities for communicating, exchanging, collaborating, and being engaged, their cognitive and behavioral abilities will remain more intact, and their quality of life could be improved.

\section{RELATED WORK}

Assistive technologies usually refer to the concept of " $\operatorname{cog}$ nitive prosthetics," i.e., compensatory strategies that alter the patient's environment, which are directed to an individual's functional skills. However, the notion of "cognitive prosthetics" mostly neglects a number of fundamental factors like motivation, personal involvement, and engagement, which are all extremely important in the treatment of people affected by dementia. In this respect, a different perspective is opened up by socially assistive robots, which are defined [3] as robots designed to provide assistance by means of social interaction.

The main purpose of these robots is to engage people in failure-free activities, thus stimulating the expression of inner emotional states, social relations, and processes of meaning negotiation. For example, some projects seek to include robots as part of the therapeutic regimen for individuals with autism [4], [5]. These studies have demonstrated that robots generate a high degree of motivation and engagement in subjects, including those unlikely or unwilling to interact socially with human therapists.

A number of studies [6]-[10] have recently reported encouraging results regarding the use of these robots in the domain of dementia care. As dementia-affected subjects suffer from a progressive cognitive and behavioral disease, which contributes to an early deterioration of the ability to interact socially, the continuous stimulation of social skills constitutes a critical issue in every therapeutic intervention in order to avoid social isolation, which is important in terms of the emergence of behavioral disorders. 


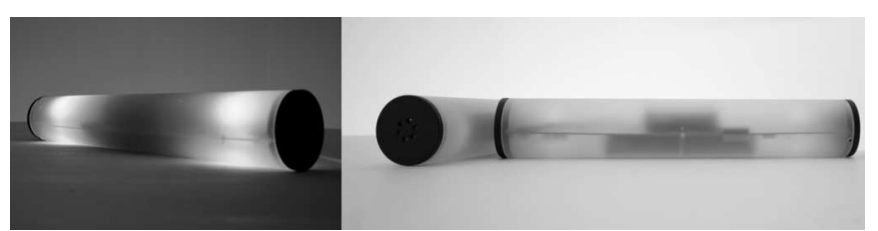

Fig. 1. Rolling pins.

\section{Modular Robotics: Rolling Pins}

Our study draws on the recent results, partly presented before, of the use of robotics in dementia care, by exploiting the opportunity offered by modular robotics [11]-[13].

Modular robots consist of a number of independent parts that can be connected in many different ways. Each unit has its own power supply and intelligence and can communicate with the others. Furthermore, each unit is able to process and communicate with its surrounding environment either through communication with neighboring robotic modules and/or through sensing or actuation [14].

Recently, we have witnessed a growing interest in modular robotics. The reason is that a robot is usually designed for one particular activity or, at best, a few closely related activities. This, of course, is not the ideal situation when the context of use requires supporting a large number of activities and meeting the needs of a heterogeneous target user group, as in the case of dementia care. In such cases, it is necessary to design either several different robots or a very complicated one.

The modular robotics approach we have adopted is, to some extent, inspired by the behavior-based robotics approach [15], although the modular robotics approach builds on the belief that behavior-based systems can include not only the coordination of primitive behaviors in terms of control units but the coordination of primitive behaviors in terms of physical control units [14], [16] as well. We can imagine a physical module being a sort of primitive behavior. Therefore, the physical organization of primitive behaviors will (together with the interaction with the environment) decide the overall behavior of the system. Hence, in a way similar to the control of robot behaviors by the coordination of primitive behaviors, we can imagine the overall behavior of a robotic artifact to emerge from the coordination of a number of physical robotic modules, with each one representing a primitive behavior.

The rolling pins (RPs) (see Fig. 1) described in this paper were designed according to the same concept as modular robotics [9], [10].

The design of the RPs has been based on the following three main characteristics.

1) Gesture-based interaction: Each time an RP is manipulated, it produces an output (visual, auditory, or tactile), both locally and remotely on the peer device, thus influencing its behavior. The therapist and the patient can, therefore, communicate by showing and imitating specific gestures with the RP and simultaneously influencing the behavior of the peer device; synchronization and coordination are key objectives of the activities supported by the RP.
2) Engagement: Patients affected with dementia refrain from exploring novel situations since they perceive their competence as not sufficient. Using the RPs, the patients are stimulated to start the exploration of the system on a "safe" and familiar base and manipulating simple objects with familiar affordances (rolling imaginary objects is one of the most frequent representation of stereotypic behavior in dementia).

3) Flexibility: Different applications of the RPs have been designed. In the "mirror" application, for example, an RP can vibrate whenever it moves at a different speed than its peer. The task of the patient is to match the therapist's rotation speed to stop the vibration. However, the therapist can choose vibration as a single feedback or to reinforce the output with a visual or aural feedback. The particularity of this task rests in its dynamic nature: The therapist can decide to slow down the rotation speed in order to help the patient in the task of synchronization or can decide to make the task more difficult to execute by deliberately challenging the synchronization or moving the pin at different speeds and rotation patterns; in other words, the therapist can adapt the task complexity during the task itself. The opportunity to continuously adapt the difficulty of the task to the skill of the patient is fundamental to the creation of an optimal experience, as well as to the maintenance the patient's attention.

In conclusion, the RPs were specifically designed to support collaborative and nonverbal exchanges between the therapist and the patient, providing them with the opportunity to establish a "pragmatic dialogue" based on visual, aural, and tactile feedback and sensory-motor interaction modalities. They embody, by design, a dialogic component that supports nonverbal communication between the therapist and the patient. They can be manipulated (e.g., grasped, rolled, and shaken), and each of these actions can produce feedback. However, the very essence of the RPs is the collaborative activity they embed. The tools communicate with each other, and by doing this, they influence each other. Each time an RP is manipulated, it produces an output (visual, auditory, or tactile) both locally and remotely on the peer device, thus influencing its behavior.

\section{A. Design Process}

In dementia care, the use of nonverbal communication is adopted widely. For example, physical contact is used in the home care ward: Nurses are trained to use affective touch to sustain and reinforce verbal instructions, especially in the case of the manifestation of behavioral disorders. In music therapy, the therapist tries to establish a dialogue by playing rhythmical instruments together with the patient or by listening to music while maintaining physical contact. Studies in music therapy [17] show a positive effect of this therapy on promoting the dialogue between the therapist and the patient by reintegrating the person within a communicative ecology-preventing isolation, regulating emotional arousal in terms of expression and inhibition, with positive implications for sufferers and caregivers, and motivating communication and participation without being speech-dependent.

These effects are achieved by utilizing the ability of gesture and hand-eye coordination to establish a dialog. 
Different studies [18] have demonstrated the importance of sensory-motor imitation in facilitating the establishment and maintenance of social relations in people with communication problems (in particular, regarding people affected with autism and dementia).

The RPs are tangible tools that can be easily manipulated just by shaking, rolling, or grasping the units. Since people suffering from dementia have several limitations in processing and understanding symbolic languages, the use of physical objects define more natural interaction modalities that can be easily understood.

In order to design interaction modalities that are meaningful for a patient suffering from dementia, a user-centered approach was adopted, including both direct observation of patients during their daily activities in the ward and extensive discussions, and interaction with the people surrounding the patients-namely, therapists, geriatrics, caregivers, and, on occasion, family members and associates. We tried to perform a nonintrusive observation of their everyday life practices, with a very naturalistic approach using only our senses and intuition and respecting the privacy of the home care guests. After each observational session, a written report was prepared and shared with doctors and care givers in order to interpret the data correctly.

Findings of the observations revealed that dementia-affected people are not motivated to participate in social activities; social interactions are very few, and they spend most of their time isolated from the others. Quite soon, we were struck by the behavioral response of these people to simple external stimuli. Very basic sensory-motor patterns, like grasping, rolling, and pulling, recurred in most of their activity. We observed some people spending hours in repeating the same basic movements, such as rolling a bottle or folding a sheet of paper. In particular, patients were generally attracted to very simple objects that show simple sensory-motor affordances and allow very basic manipulation.

In parallel with the observation of the elderly people in the ward, an extensive analysis of their residual abilities was conducted to define a conceptual framework for understanding the progressive manifestation of cognitive and behavioral symptoms. Even if the degree and the temporal manifestation of the impairment are different for each individual patient, some features can be commonly observed. For example, the first symptoms to appear are episodic memory deficits and the related difficulties in remembering recent events. Furthermore, since dementia-affected patients lose the ability to retain and process complex stimuli they experience increasing difficulty in making sense of the external world. Any system/technology/support, to be successfully integrated in the treatment of dementia, should strictly address the following requirements. First, the grossmotor physical limitations of these patients suggest the need for interaction with objects whose dimensions and weights are suitable for an easy manipulation (technical details on weight and dimensions of the RPs are provided in Section III-B). Because of their difficulties in making sense of novel and complex situations, it is fundamental to design very simple and clear interaction modalities based on physical and sensorial manipulation (rolling and shaking are the main interaction modalities

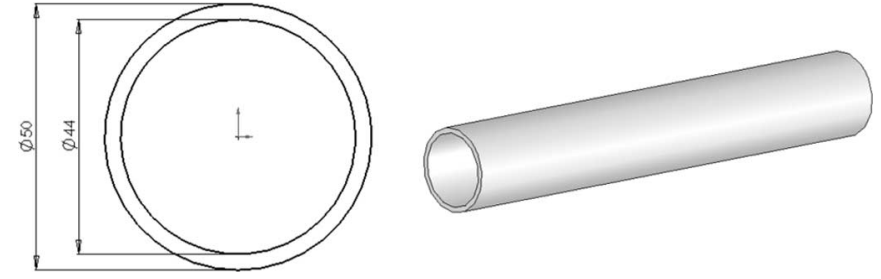

Fig. 2. CAD drawing of tube.
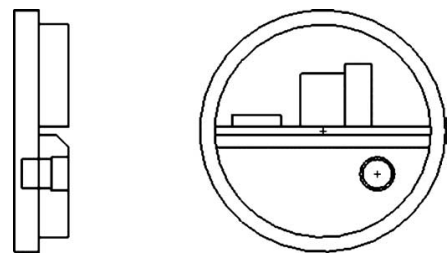

Fig. 3. Right end cap (seen from PCB mounting side).

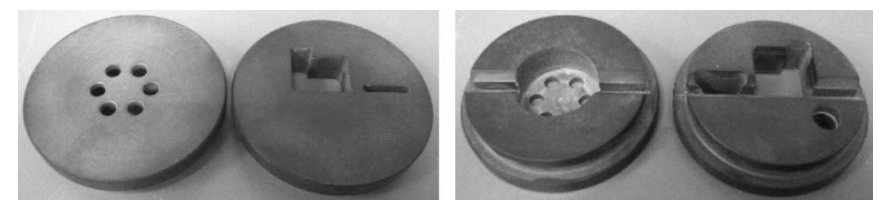

Fig. 4. End caps (seen from the (left) outside and (right) inside).

with the RPs). Next, in order to exploit residual abilities related to procedural knowledge, the system should be able to stimulate familiar sensory-motor patterns (e.g., rolling is a very familiar sensory-motor pattern for these subjects). Also, other stimuli should be reduced in order to avoid the dispersion of the limited attention span of patients (stimuli are selected and controlled by the therapist). Finally, due to the difficulties that patients affected by dementia have with verbal communication, the system should sustain nonverbal dialogs [18] (the RPs sustain a gesture-based dialogue).

The results of the observations and studies of clinical cases and therapeutic practices were used as design guidelines for the new system. The process generated a number of concepts that were continuously assessed by therapists and physicians, resulting in the selection of one concept, known as the RPs, for its potential to evoke consolidated sensory-motor patterns, to enable coordination and communication without verbal exchanges and generate an intrinsic motivation to actively participate in a social exchange.

\section{B. Technology Design}

An RP consists of a semitransparent plastic tube (see Fig. 2) with solid end caps (see Figs. 3 and 4). All the electronic components are placed on one large printed circuit board (PCB) inside the tube (see Fig. 5). The total weight of an RP is $350 \mathrm{~g}$, including batteries.

The RP has three types of feedback: red, green, and blue (RGB) light, sound, and vibration. Furthermore, RPs are able to communicate with each other or with similar devices equipped with the same radio communication technology. The RPs are 


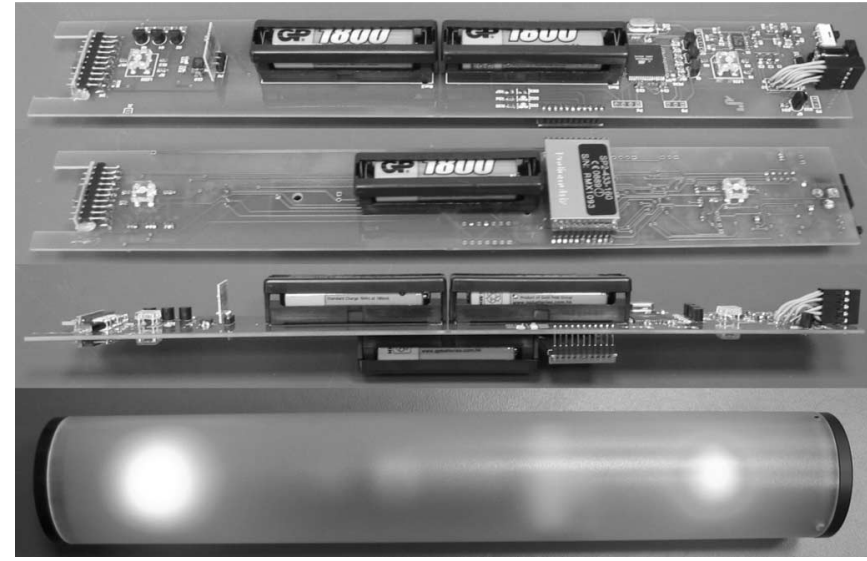

Fig. 5. PCB seen from the top, the bottom, the side, and an assembled RP.

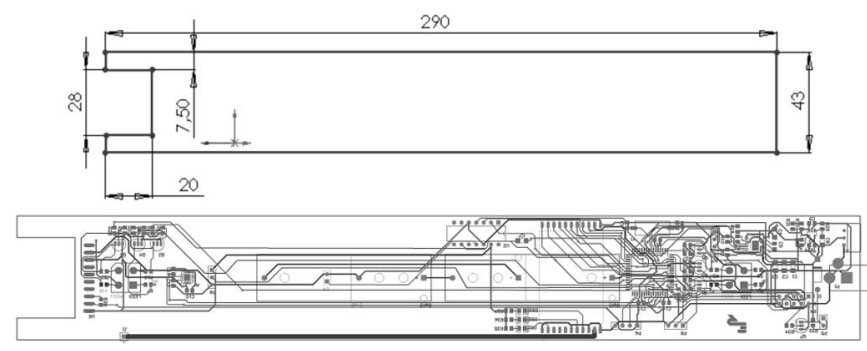

Fig. 6. (Top) Dimensions of the PCB (thickness $1.6 \mathrm{~mm}$ ). (Bottom) PCB layout (top layer in front).

capable of measuring their orientation and the speed of their rotation [19].

The outer tube of the RP (see Fig. 2) has the following dimensions: outer diameter, $50 \mathrm{~mm}$; inner diameter, $44 \mathrm{~mm}$; length, $290 \mathrm{~mm}$; weight, $150 \mathrm{~g}$.

The right end of the tube is equipped with three holes with small screws for holding the right end cap in place. The left end cap is glued to the tube. The end caps (see Figs. 3 and 4) have the following properties: outer diameter, $50 \mathrm{~mm}$; length, $11 \mathrm{~mm}$; weight (each), $22 \mathrm{~g}$; material, sandblasted black plastic.

The left end cap is equipped with six holes $(\phi=4 \mathrm{~mm})$ for the sound to travel through, and a countersink that holds the loudspeaker (see Fig. 4). The right end cap is equipped with three holes to hold the charge connector, Joint Test Action Group (JTAG) programming interface, and the ON/OFF switch (see Fig. 4). Furthermore, the right end cap carries the vibrator. The caps are also equipped with countersinks that hold the PCB in place (see Figs. 3 and 4).

The electronic parts of the RP consist of one large PCB (see Figs. 5 and 6) and an internally connected loud speaker that is mounted on the left end cap. The weight of the PCB is 78 or $156 \mathrm{~g}$, with batteries installed.

Besides standard components such as resistors, capacitors, and diodes, the PCB carries the following electronic components.

\section{1) Computing}

a) The RP is controlled by a microcontroller from Atmel (ATmega128 running at $4 \mathrm{MHz}$ ).

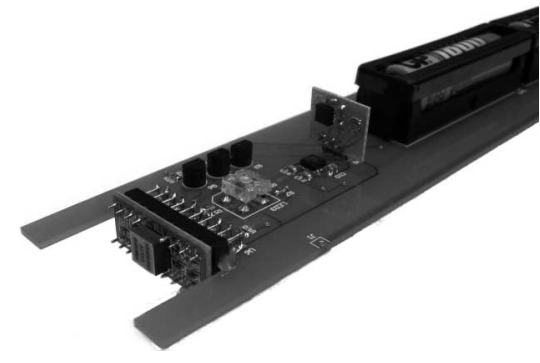

Fig. 7. Gyro and accelerometer end of the PCB.

2) Sensors

a) A gyroscope from Analog Devices (ADXRS300EB) is used to measure the speed and direction of the rotation (see Fig. 7).

b) Two 2-axis accelerometers from Analog Devices (ADXL320) are used for measuring the orientation of the RP. One of the accelerometers is mounted vertically on the PCB; therefore, all three axes can be measured (see Fig. 7).

3) Actuators

a) Four RGB LEDs from Dotlight (SRGB7130 Superflux LED $130^{\circ}$ ) are used for visual user feedback. The four LEDs are divided into two pairs (one pair in each end) that are individually controllable (see Fig. 5). It is possible to generate any color due to the nature of the RGB LEDs.

b) One vibrator (from a mobile phone vibrator unit) is used for sense-of-touch user feedback. The vibrator is not shown in the figures.

c) One loudspeaker (ELFA 30-204-84, $20 \mathrm{~mm}$, $100 \Omega$ ) is used for audible user feedback. Any hearable sound can be generated or combined to form short melodies. The loudspeaker is not shown in the figures.

d) One digital volume adjuster (Dallas DS1666-010) for the adjustment of the volume of the loudspeaker. (This device is not installed in the RPs used in the experiments presented here due to limitations in the control of the volume.)

\section{4) Communication}

a) One communication module (RadioMetrix SP2 433 160) is used for communication with other RPs or similar devices equipped with the same communication technology (see Fig. 5).

5) Power:

a) three $\mathrm{AA}$ rechargeable batteries (GP NiMH $1800 \mathrm{~mA} \cdot \mathrm{h}$ );

b) one regulated $5-\mathrm{V}$ 300-mA charge pump (TI TPS60130 3× AA to $5 \mathrm{~V}$ );

c) one low-power shutdown circuit (MAX834) for shutting down the power supply to the electronic circuitry when the battery supply voltage goes below a fixed voltage level.

6) External connectors:

a) ON/OFF switch (see Fig. 8, right); 


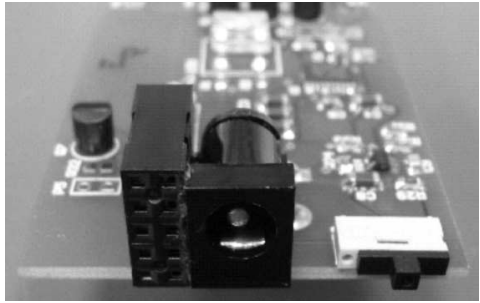

Fig. 8. Connector end of the PCB.
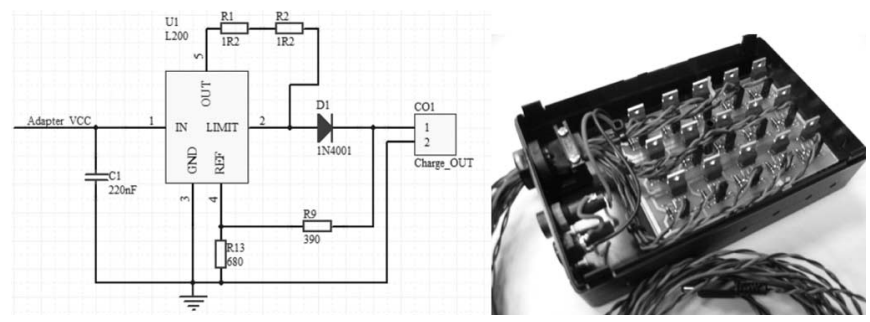

Fig. 9. (Left) Schematics of one unit charger. (Right) 15-unit charger.

b) JTAG programming interface (for programming of the microcontroller) (see Fig. 8, left);

c) charge connector (for use with customized external charger). When a charge plug is placed on the charge connector, the circuitry is disabled, and only the connection to the batteries is maintained (see Fig. 8, center).

Each RP unit is equipped with three AA NiMH rechargeable batteries. The batteries are manufactured by GP batteries, the nominal voltage of each battery is $1.2 \mathrm{~V}$, and the capacity is $1800 \mathrm{~mA} \cdot \mathrm{h}$. The batteries have no memory effect and have up to 1000 recharge cycles. The batteries are charged while in the units; therefore, an external constant-current charger is used to charge the batteries. The charger can charge 15 units at a time, and each unit is charged with a constant current of approximately $177 \mathrm{~mA}$. The relatively low charge current results in a recharging time of approximately $10 \mathrm{~h}$ when the batteries are totally drained, but the typical charge time is less than that. Note that the charger is specialized for charging of three NiMH rechargeable batteries in series and not for common use. The charge voltage is adjusted to approximately $4.36 \mathrm{~V}$, where the maximum allowable charge voltage is $4.5 \mathrm{~V}$.

The reason for choosing a relatively slow constant-current charger instead of an intelligent fast charger is a tradeoff between complexity and charging time. An intelligent fast charger requires rather complex circuitry and often requires additional cooling. A constant-current charger only requires simple circuitry and, for safety reasons, such as to avoid overheating, the charge current should be $1 / 10 \mathrm{C}$. The schematics of one charger and a photograph of the 15-unit charger are shown in Fig. 9.

The charger is supplied by a 7.5-V dc 3.25-A power supply, but the charger uses only approximately $1.5 \mathrm{~A}$ when all 15 charging units are in use. For safety reasons, the 15-unit charger has a 2.5-A fast fuse installed. A small green LED installed in the lid indicates whether or not the charger is supplied from its

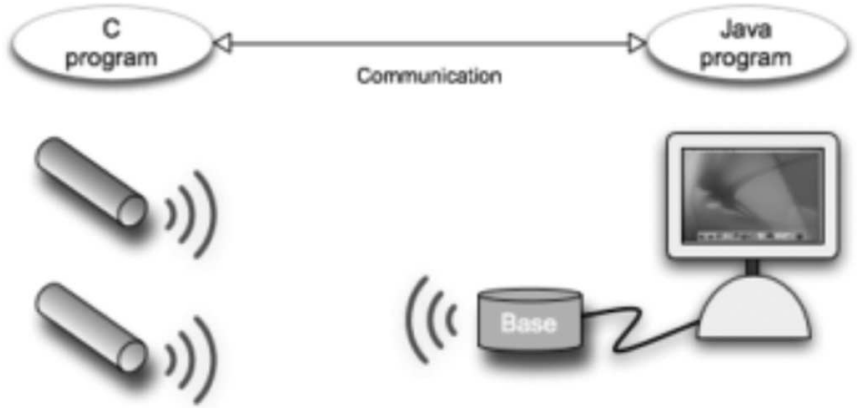

Fig. 10. Software framework.

external power supply or whether or not the fuse is intact $(3 \mathrm{~mm}$ green LED with $270 \Omega$ resistor in series).

The output of each charger goes to a 2.1-mm dc Jack connector (note that ground is connected to the inner part of the pin).

A base unit is connected to an ordinary PC via a serial (RS232) connection. It is used for the control and settings of the adjustable parameters of the entire system. It can be used for runtime control of the RPs, but in the study presented here, it is used only a priori to download application parameters to the RPs. Furthermore, the base unit can control a specialized external dimmer to control the surrounding environment via pulsewidth modulation (PWM) (adjustment of the light intensity of externally connected colored light lamps). This option is not used in the experiments presented here. The base unit contains a standard main PCB and a radio module. The radio module communicates with the corresponding radio module in the RPs.

\section{Software Design for the RPs}

The RPs are generally used in pairs, since the local feedback of each RP can be dynamically set depending not only on its own speed and orientation but on the speed and the orientation of the peer RP as well. For example, the local feedback of each RP can be a function of the sum of the rotation speed of both RPs; potentially, it can be a function of any other operation between the speed of the rotations (and orientations) of two RPs. Furthermore, since each RP runs its own software, each of them can generate its own feedback in relation to rules that are different from the rules of the other one.

The software framework for the RPs allows the applications to run autonomously in the objects, while also providing the possibility for communication with a host PC. The PC software is responsible for the control of the application selection, thus allowing the therapist to select an application for usage. Hence, there is a PC software and an embedded RP software, as shown in Fig. 10.

Since the PC is used for application selection only, this implies that the application itself is controlled in a distributed manner by the objects. Each object contains no identification, thus enabling maximum freedom in the assembly — each RP can be replaced by another RP. The advantages of this are that a program can run without the presence of the $\mathrm{PC}$, the same program code can be 


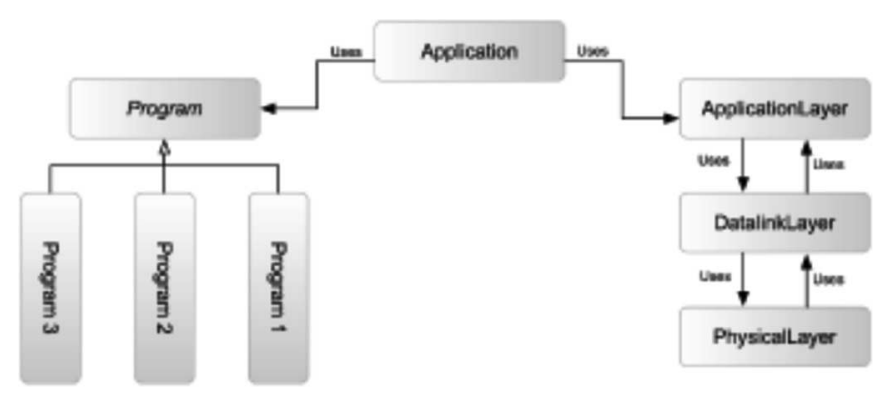

Fig. 11. PC software architecture.

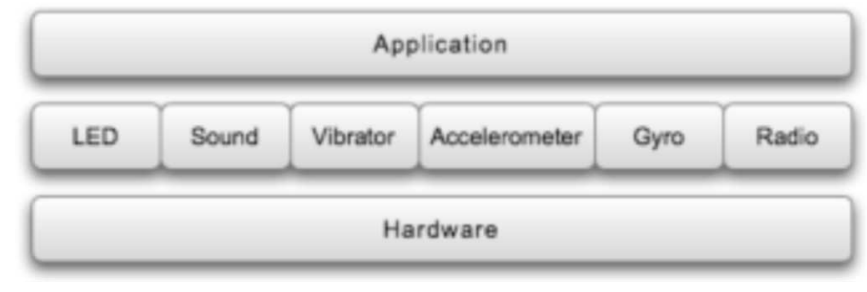

Fig. 12. RP control layers.

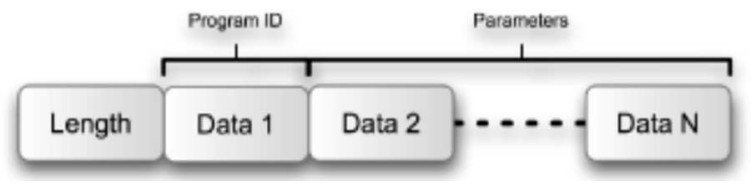

Fig. 13. Transmission package structure.

loaded into all RPs, the program can be made independent of the number of pins, less wireless radio communication is needed, and there will be faster information flow.

For the ease of the application development, a framework was constructed on the PC side in JAVA. Thereby, the developer only adds his/her program by respecting a contract settled by the framework. In the objects (cylinders and pins), a C library was constructed to provide access points to the different hardware functionalities of the objects.

The software architecture on the PC side is shown in Fig. 11.

The application developer inherits from the program interface and implements all the necessary methods. The application must then be implemented on the RP. This is done by implementing the application logic in the RP on top of the library, which is illustrated in Fig. 12.

The library consists of a driver layer that provides ease of use for the underlying hardware. At the current hardware state, an RP can communicate through two different channels- RS232 (standard serial) or wireless. The communication protocol for each of them is described in the following paragraphs.

The transmission package structure is illustrated in Fig. 13. The first byte is always the length of the package, and the rest (maximum 254 bytes) are data fields. This implies that the application layers must agree on a common communication protocol. Fig. 13 illustrates that the first data field could be used as a program ID and the rest as parameters.

The PC side of the application consists of an easy-to-use GUI, which has the capability of plugging in the different applications that abide by the program interface.

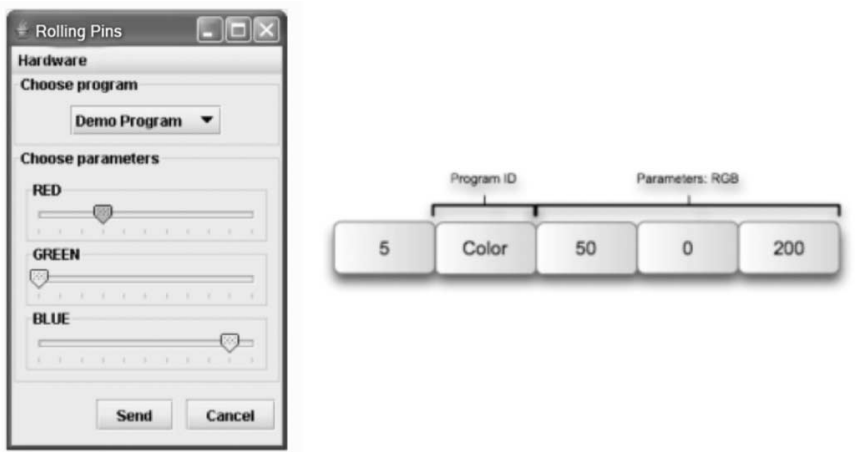

Fig. 14. (Left) Simple example GUI on the PC for changing the colors. (Right) Transmission package produced from setting the color in the GUI above.

A simple example could be a program that changes the color intensity for RGB. This example of a simple GUI is illustrated in Fig. 14. The GUI was designed to enable the therapist to modify the sensorial stimuli by selecting different combinations of visual, aural, and tactile feedback. The tactile stimulation can be produced either by the physical surface of the RP (on which different types of covers can be placed) or generated by the vibration actuator.

Furthermore, the therapist can also select different communication rules between the RPs. To summarize, the therapist can adapt the complexity of the interaction to the specific needs of each patient, the therapeutic objectives, and the specific therapeutic protocol.

In the aforementioned example, the user can set the intensities of the RGB LEDS. This information is collected in a transmission package, as shown in Fig. 14 (right), and sent from the PC to the RP. When the RP receives the message, it checks the program ID field and reacts accordingly.

The software implementation is open to different strategies; the application at hand can be implemented with a centralized control or distributed control. Furthermore, the user can choose to implement different communication strategies, e.g., connection-oriented protocol or connectionless protocol.

\section{FIELD TRIALS}

The main claim of this paper is that the dialogic component embedded in the RPs constitutes an added value in nonverbal communication, thus enabling the therapist to establish different levels of exchanges beyond verbal communication. In other words, the RPs create a shared interaction space in which the therapist has a number of different opportunities to influence the patient's behavior by showing specific sensory-motor interaction patterns with the RP and simultaneously affecting the feedback of the RP held by the patient. We do indeed believe that the dialogic component plays a critical role in mediating the communication between the therapist and the patient, thus favoring the spontaneous engagement of the patient in the activity and enabling basic forms of nonverbal communication, such as imitation and coordination. In order to assess if this particular nonverbal and gesture-based exchange can sustain effective communication and coordination between the therapist and the 
patient, we designed an experiment to compare the use of the RPs under two conditions:

1) with the RPs used as independent devices, interactive but not communicating with each other (individual modality);

2) with the RPs communicating with each other (dialogic modality).

Specifically, we defined three hypotheses.

1) The first hypothesis concerns the capability of the RPs to activate consolidated sensory-motor patterns in the procedural memory, thereby encouraging dementia-affected people to participate in the activity: Subjects working in the dialogic modality are more inclined to spontaneously engage in interaction with the RPs than subjects working in the individual modality.

2) The second hypothesis addresses the role of the dialogic modality in stimulating sensory-motor coordination: Subjects working in the dialogic modality establish a more stable and solid sensory-motor coordination with the therapist than subjects working in the individual modality.

3) The third hypothesis concerns the patient's involvement and engagement in the activity: Subjects working in the dialogic modality manifest a stronger intrinsic motivation to participate in the activity than patients working in the individual modality.

\section{A. Software Application}

Two programs have been defined for the experiment: the program used in the individual modality and the program used in the dialogic modality.

In both the programs, the input to the system consisted of the shift frequency of the RPs. The shift frequency was calculated by the number of times the RP changed the direction of rotation within a given interval of time (1 s). In other words, the shift frequency depends on how fast the user rotates the RP forward and backward. Since this information is provided by the gyro, the detection of the shift frequency can be done independently of the knowledge of the orientation of the RPs: This allows the users (both the therapist and the patients) to try different kinds of manipulation. However, in the experiment, the whole activity was carried out over a table.

In the program used for the individual modality, four different shift frequency ranges have been identified. When the shift frequency of the RP is within the boundaries of a certain range, it provides specific visual and aural feedback. In particular, the color blue and the tone $\mathrm{C} 4$ are associated with the lower values of the shift frequency (see Table I, range A), while the color red and the tone B4 are associated with the higher values of the shift frequency (see Table I, range D). The colors green and orange have been associated with the intermediate ranges (see Table I). The shift frequency ranges have been defined in collaboration with therapists and physicians in order to assure that the patients have the physical skills to produce the required movements and the cognitive skills to discriminate among them.

The choice of the colors has been based on two considerations. First of all, we have exploited the natural mapping between cold colors (e.g., blue) and the impression of calmness
TABLE I

INDIVIDUAL MODALITY: INPUT/OUTPUT RELATION

\begin{tabular}{llc}
\hline Shift Frequency Ranges & Colour & Sound \\
\hline A & Blue $(0,0,255)$ & C4 \\
B & Green $(0,255,0)$ & E4 \\
C & Orange $(255,128,0)$ & G4 \\
D & Red $(255,0,0)$ & B4 \\
\hline
\end{tabular}

The values of the RGB parameters are in brackets. In the sound column, tones have been reported adopting the letter notation.

TABLE II

DIALOGIC MODALITY: INPUT/OUTPUT RELATIONSHIP

\begin{tabular}{clll}
\hline \hline $\begin{array}{l}\text { Shift Frequency } \\
\text { Ranges } \\
\text { (RP1 + RP2) }\end{array}$ & Colour & Vibration & Sound \\
\hline A & Blue $(0,0,255)$ & No & C4 \\
B & Blue/Green $(0,128,128)$ & No & E4 \\
C & Green $(0,255,0)$ & No & G4 \\
D & Green/Yellow $(128,255,0)$ & No & B4 \\
E & Yellow $(255,255,0)$ & No & C5 \\
F & Yellow/Orange $(255,192,0)$ & No & E5 \\
G & Orange $(255,128,0)$ & No & G5 \\
H & Orange/Red $(255,64,255)$ & No & B5 \\
I & Red $(255,0,0)$ & Yes & C5 \\
\hline The RGB parameters are in brackets. In the sound column, notes have been reported adopting
\end{tabular}

the letter notation.

and between warm colors (e.g., red) and the impression of dynamism [20]. We have associated the lower values of the shift frequency with cold colors (blue and green), and as the shift frequency increases, the associated colors progressively move toward warmer tonalities (orange and red). Second, in order to maximize the contrast between the presented colors and to facilitate elderly people in recognizing them, we selected two primary colors (red and blue) and their complements (green and orange). Also, in the case of sound, we have relied on the natural mapping between lower tones and calm movements and shrill tones and quick movements. In particular, in order to have a harmonic progression, we have chosen the first, the third, the fifth, and the seventh degree of the $\mathrm{C}$ major scale.

In the dialogic modality, the two RPs communicate with each other; in the specific implementation adopted for the experiment, the local feedback of each RP is given by the sum of the shift frequencies of the peers.

Table II shows the association between the shift frequency ranges used in the program for the dialogic modality and the visual, aural, and tactile feedback generated by each RP.

For example, whenever the sum of the shift frequencies of two communicating RPs is within range $\mathrm{A}$, each peer will show the color blue and will generate the tone $\mathrm{C} 4$. As the sum of the shift frequencies increases, the color changes from cold to warmer tonalities, and the tones become higher; when it reaches the highest value, both RPs start vibrating to reinforce the feedback. As it is possible to observe in Table II, in the program used in the dialogic modality, a new set of shift frequency ranges has been added (from $\mathrm{E}$ to I). This was done to discriminate 

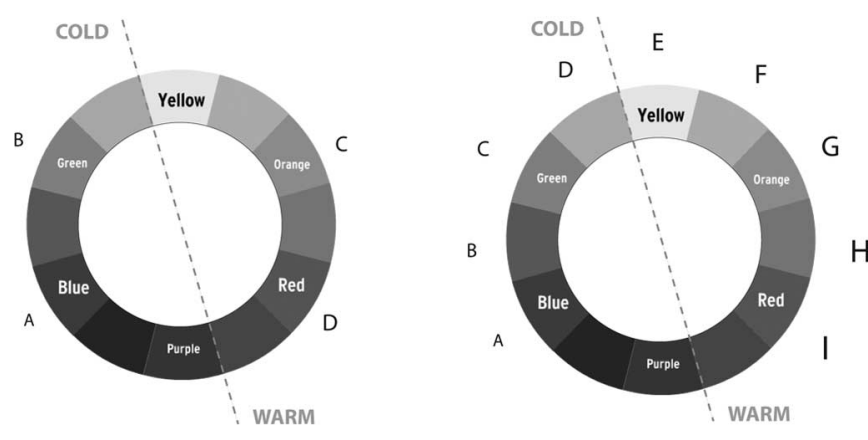

Fig. 15. Mapping of shift frequency ranges and colors (left) in the individual modality and (right) in the dialogic modality.

between the different values resulting from the sum of the shift frequencies of each RP. The colors and the associated sounds have been selected following the same considerations made for the individual modality program.

In Fig. 15, it is possible to observe the difference between the two programs in terms of mapping between shift frequency ranges and colors. It is important to note that the full range of feedback can only be experienced if both the therapist and the patient are involved in the activity.

In fact, if the therapist does not move his/her own RP, the patient can only reach the shift frequency values from range $\mathrm{A}$ to range $\mathrm{E} / \mathrm{F}$.

\section{B. Experimental Plan}

A between-subject experiment with two independent groups was designed. One of the two groups interacted with the system set in the dialogic modality (dialogic condition) and the other one with the system set in the individual modality (individual condition). Both groups performed the whole experimental protocol (phases A, B, and C-see Section IV-C). In the dialogic modality, the two RPs communicate with each other: In the specific implementation adopted for the experiment, the local feedback of each RP is given by the sum of the rolling speeds of the peers. In the individual modality, the RPs do not communicate with each other: The RP local feedback only depends on its own rolling speed. This modality of interaction constituted the baseline against which the subjects' performance resulting in the dialogic modality was compared.

The experiment was conducted in the nursing home "Casa Protetta Albesani," which is an institution located in northern Italy (Castel S. Giovanni, Piacenza, Italy) that provides hospitality to 150 elderly people with different degrees of cognitive and behavioral diseases. The experimental plan and the entire project were submitted to the Ethical Board of the Home Care institution and approved by all members. Therapists and geriatric physicians discussed the methodology and proposed a specific protocol to conduct the experiment and sampling the subjects. They later followed all of the phases of the experiment and collaborated in the final interpretation and communication of the results. Also, the relatives of the elderly people participating in the study were involved in the conception of the experiment and were constantly informed about the evolution of the ther-
TABLE III

EXPERIMENTAL GROUP

\begin{tabular}{lccc}
\hline \hline System modality & $\mathrm{N}$ & Age & MMSE \\
\hline Dialogic & 6 & $76.2(14.4)$ & $20.5(3.3)$ \\
Individual & 6 & $86.3(9.2)$ & $21.5(4.7)$ \\
\hline
\end{tabular}

The number of subjects $(N)$, the mean age (age), and the mean MMSE (MMSE) are shown for each experimental condition. Standard deviations have been reported in brackets. The MMSE is a test used to evaluate the cognitive impairments of dementia-affected patients (0-15: severe; 15-24: moderate; 24-30: mild/normal). The age difference between the two groups can be explained by the presence of a younger subject (56) in the group working with the system set in the dialogic modality. Physicians and therapists considered that the cognitive impairment of this subject can be assimilated with the other subjects. His MMSE score (20) confirmed this.

apy. Together with physicians and therapists, 12 patients were sampled with a mini mental state examination (MMSE) [21], resulting in scores in the range of 16-27. Each patient was randomly assigned an experimental group: we obtained two equally numbered groups (see Table III).

All subjects working in the two experimental conditions followed the same protocol composed of three phases (as detailed in Section IV-C). Each phase was designed to test a specific hypothesis: phase A to test hypothesis 1, phase B to test hypothesis 2 , and phase $\mathrm{C}$ to test hypothesis 3 . Therefore, in each phase, different behavioral indicators have been selected in order to evaluate the subjects' performance (for details, see Section IV-D).

\section{Experimental Protocol}

The experimental setting was the same for the two groups. The therapist and the patient sat down around a table one in front of the other. An RP was placed on patient's side and another one on the therapist's side. The experimental protocol was composed of three phases and structured as shown in Table IV. Each phase was specifically designed to test one of the three hypotheses.

Phase A was designed to help clarify if the dialogic modality stimulates the patient's autonomous initiative to participate in the activity, without any additional instruction from the therapist (hypothesis 1). For this reason, the therapist was invited to pronounce a neutral sentence to initiate the activity.

Phase B was designed to observe the patient's behavior in a coordination activity, in order to test hypothesis 2 . The main difference between the two phases is that while in phase A, the therapist generates discrete interaction patterns with the RP, waiting for the patient's answer, and in phase B, he/she interacts in a continuous manner with the RP explicitly inviting the patient to join the activity.

At the end of the session, the patients were asked to answer a standard version of the Intrinsic Motivation Inventory (IMI) [22] in order to test hypothesis 3 (phase C).

\section{Coding and Analysis}

Each experimental trial was video-recorded. The camcorder was placed on a tripod and positioned in such a way that it was possible to record the subjects' expression, and the therapist's and subjects' hands manipulating the RP. However, the video camera was not visible to the subjects. Two experimenters carried out the video analysis with the support of a specific software (The Observer XT). 
TABLE IV

EXPERIMENTAL PROTOCOL

\begin{tabular}{|c|c|}
\hline & PHASE A \\
\hline Description & $\begin{array}{l}\text { Therapist repeats every single interaction pattern } 3 \text { times. } \\
\text { At the end of each repetition, he/she waits }(5-10 \text { seconds) } \\
\text { for the patient's reaction. The therapist initializes the } \\
\text { phase saying: "let's see how these objects work". }\end{array}$ \\
\hline Patterns & $\begin{array}{l}\text { - Rolling the pin slowly over a short distance (from } 5 \mathrm{~cm} \\
\text { to } 10 \mathrm{~cm} \text { ) } \\
\text { - Rolling the pin slowly over a long distance (from } 15 \text { to } \\
30 \mathrm{~cm} \text { ) } \\
\text { - Rolling the pin fast over a short distance (from } 5 \text { to } 10 \\
\mathrm{~cm} \text { ) } \\
\text { - Rolling the pin fast over a long distance (from } 15 \text { to } \\
30 \mathrm{~cm} \text { ) }\end{array}$ \\
\hline & PHASE B \\
\hline
\end{tabular}

The therapist produces the interaction patterns described
below. Each pattern lasts about 30 seconds (except the last
one which depends on the therapist's feeling about the
situation). The therapist initiates the phase saying "let's
do this together".

Patterns The same patterns as in phase A

PHASE C

IMI administration. After the activity, the therapist administers the Intrinsic Motivation Inventory.

For phases A and B, a number of different behavioral indicators were defined and observed in order to verify the first and the second experimental hypotheses. Generally speaking, two kinds of behavioral indicators were defined: 1) discrete indicators codifying events whose duration is not relevant and 2) continuous indicators codifying events with a clear start and end. In the latter case, the video data from each and every trial of a given patient were segmented into 1-s intervals; the patients' activities were coded by scoring the continuous behavioral indicators every second of the trial. The scores for each trial were then summed up, thus yielding the total number of occurrences of each behavior during a specific trial and the total amount of time the patient was engaged in each behavior during that trial [9].

In order to test hypothesis 1 , we defined a discrete behavioral indicator (called answer) and observed its occurrence during phase A: We scored the number of times the patient reproduced the interaction patterns proposed by the therapist with his/her RP.

In order to test hypothesis 2 , during phase $\mathrm{B}$, we codified the patient's behavior in relation to three continuous behavioral indicators.

1) The patient produces no interaction pattern while the therapist interacts with the RP (none).

2) The patient does not reproduce the therapist's interaction patterns but generates them randomly (random).

3) The patient simultaneously reproduces the same interaction patterns as the therapist (tuning).

In order to verify hypothesis 3, we administered the IMI [22]. This is a multidimensional measurement questionnaire
TABLE V

MeAn Lengths of Phases A AND B

\begin{tabular}{lll}
\hline & Phase A & Phase B \\
\hline System modality & & \\
\hline Dialogic Modality & $258.5(85)$ & $235.7(70)$ \\
Individual Modality & $234.7(130)$ & $244.6(46.8)$ \\
\hline
\end{tabular}

The table details the mean lengths (and the standard deviation in brackets) of phases A and B for each experimental group. Means and standard deviation are expressed in seconds.

that assesses participants' subjective experience related to a target activity. The questionnaire assesses participants' interest/enjoyment, perceived competence, effort, value/usefulness, felt pressure and tension, and perceived choice while performing a given activity, thus yielding six subscale scores.

Subscales are rarely used all together; a number of subscales are usually selected in relation to the experimental objectives. In our experiment, we used a questionnaire composed of three subscales: interest/enjoyment, perceived competence, and pressure/tension [22].

\section{RESUlts}

Phases $\mathrm{A}$ and $\mathrm{B}$ in the two conditions lasted about $4 \mathrm{~min}$. Table $\mathrm{V}$ details the mean lengths (and the standard deviation in brackets) of the two phases for each experimental group. Phase $\mathrm{C}$ (the administration of IMI) lasted about $5 \mathrm{~min}$. Also, considering the initial 5 min when patients familiarized themselves with the environment, each experimental trial lasted about $20 \mathrm{~min}$.

\section{A. Hypothesis 1}

An analysis of the answer behavioral indicator was conducted on the data related to the video analysis of phase A. The independent variable was the system modality, dialogic, or individual. The dependent variable was the number of occurrences of the answer discrete behavioral indicator.

In both conditions (individual and dialogic), subjects either reproduced each interaction pattern proposed by the therapist (12), or they ignored all of them. Every subject but one (who reproduced 11 interaction patterns out of 12) working in the individual condition did not autonomously reproduce any interaction pattern proposed by the therapist.

Instead, in the dialogic condition, four subjects out six reproduced every interaction pattern proposed by the therapist.

In Table VI, means and standard deviation of the two groups have been reported; on average, subjects working in the dialogic condition reproduced a larger number of interaction patterns than subjects in the individual condition.

As a result of the nonnormal distribution of the "Answer" variable, a nonparametric test was applied (Mann-Whitney U): The difference of the means appears to be statistically significant $\left(U=7, z=-2.008,{ }^{*} p=0.045\right)$.

\section{B. Hypothesis 2}

A two-way $(2 \times 3)$ mixed analysis of variance (ANOVA) was conducted to examine the differences between the two 
TABLE VI

PHASE A RESUlTs

\begin{tabular}{lll}
\hline & $\begin{array}{l}\text { Dialogic } \\
\mathrm{N}=6\end{array}$ & $\begin{array}{l}\text { Individual } \\
\mathrm{N}=6\end{array}$ \\
\hline Answer & & \\
\hline Mean & 8.00 & 1.83 \\
st.dev & 6.20 & 4.49 \\
difference & 6.17 & \\
Mann-Whitney $\mathrm{U}$ & $7 *(\mathrm{p}=0.045)$ & \\
\hline
\end{tabular}

Results of phase A are shown: for each experimental condition, the mean of the "Answer" has been reported. The reported values indicate on average the number of interaction patterns that the patients reprodued with his/her RP. Furthermore, the standard deviations, the difference between means (difference), and the statistical test have been reported.

TABLE VII

PHASE B ANOVA TABLE

\begin{tabular}{llll}
\hline \hline & None & Random & Tuning \\
\hline \multirow{2}{*}{ Dialogic $(\mathrm{N}=6)$} & 5.35 & 41.11 & 192.42 \\
& $(6.29)$ & $(58.26)$ & $(57.949)$ \\
Individual $(\mathrm{N}=6)$ & 41.00 & 95.46 & 98.17 \\
& $(45.16)$ & $(50.62)$ & $(41.40)$
\end{tabular}

The means and standard deviation (in brackets) are shown for each experimental condition in relation to none, random, and tuning behavioral indicators. Means and standard deviation are expressed in seconds.

experimental groups, which were codified during phase B according to three behavioral indicators (none, random, and tuning). The independent variables included a "between groups" variable, the system modality (individual and dialogic), and a "within subject" variable articulated on three levels (none, random, and tuning).

The dependent variable was the total amount of the occurrences of each behavioral indicator analyzed in the 1-s video segments recording the entire duration of phase B of each trial. As stated in Section IV-D, this value corresponds to the amount of time the patient spent on performing each single behavioral indicator.

The results of phase B have been reported in Table VII.

On average, the duration of none and random behavioral indicators was longer in the individual condition than in the dialogic, while the duration of the tuning behavioral indicator was longer in the dialogic condition than in the individual one.

Furthermore, as Fig. 16 shows, in the dialogic condition, there was a clear difference between duration means of tuning and random (151.31 s) and between duration means of tuning and none $(186.07 \mathrm{~s})$, while the difference between duration means of random and none was less evident (35.76 s).

In the individual condition, the difference between the behavioral indicators means was less considerable than in the dialogic condition (the difference between duration means of tuning and random was $2.61 \mathrm{~s}$; the difference between duration means of tuning and none was $57.17 \mathrm{~s}$; the difference between duration means of random and none was $35.76 \mathrm{~s}$ ).

There was a significant interaction between the system modalities (individual or dialogic) and the behavioral indicators (none, random, and tuning $\left(\mathrm{F} 2.30=8.802,{ }^{* * *} p=0.001\right)$.

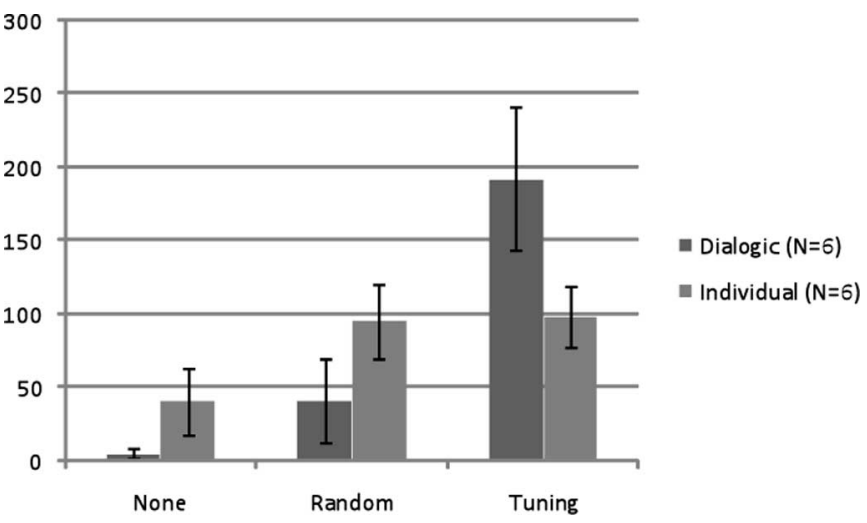

Fig. 16. Graph representing mean values of each behavioral indicator (none, random, and tuning) during phase $\mathrm{B}$, both concerning the individual modality and the dialogic modality. Mean values are expressed in seconds.

Simple main effect analysis shows that the duration of the dialogic and individual groups' behavioral indicators was significantly different concerning tuning $\left(\mathrm{F} 1.10=10.509,{ }^{* *} p=\right.$ $0.009)$ but not concerning none $(\mathrm{F} 1,10=3.666, p=0.085)$ and random $(\mathrm{F} 1.10=2.974, p=0.115)$.

The simple main effect of the behavioral indicators was significant for the dialogic group $\left(\mathrm{F} 2.15=26.136,{ }^{* * *} p=0.000\right)$ but not for the individual group $(\mathrm{F} 2.15=3.076, p=0.076)$. Post hoc Tukey tests (at $p=0.05$ ) were conducted to further explore this effect. For the simple main effect of the behavioral indicators for the dialogic group, the duration was significantly different between none and tuning and between random and tuning. However, the duration of none and random was not significantly different.

These results clearly indicate that in the dialogic condition, the subjects are tuned in with the therapist for a longer amount of time than in the individual condition; furthermore, while the subjects in the dialogic condition mainly performed the same interaction patterns of the therapist (tuning), the subjects in the individual condition performed the none, random, and tuning behaviors indifferently, without any statistically significant difference.

\section{Hypothesis 3}

An analysis of the intrinsic motivation has been performed on the data collected from the administration of the IMI.

The independent variable was the "groups variable" system modality (dialogic or individual). The scores obtained by the subjects on the three subscales adopted in the IMI (interest/enjoyment, perceived competence, and tension) were treated as distinguished dependent variables. As shown in Table VIII, there was a positive trend from the dialogic to the individual modality for the interest/enjoyment and perceived competence scales: The means of the interest/enjoyment and perceived competence scores are higher in the dialogic condition than in the individual one. The means of the tension score were higher in the dialogic condition than in the individual one. Since tension in the IMI questionnaire is considered as a negative predictor of intrinsic motivation, there was a slightly negative trend 
TABLE VIII

PHASE C IMI

\begin{tabular}{lll}
\hline & $\begin{array}{l}\text { Dialogic } \\
\mathrm{N}=5\end{array}$ & $\begin{array}{l}\text { Individual } \\
\mathrm{N}=6\end{array}$ \\
\hline Interest/Enjoyment & & \\
\hline Mean & 6.4033 & 5.4 \\
st.dev & 0.4755 & 1.1606 \\
Difference & 1.0033 & \\
Unpaired T-Test & $1.95 *(\mathrm{p}=0.041)$ \\
\hline Tension & \multicolumn{2}{|}{} \\
\hline Mean & 4.75 & 4.55 \\
st.dev & 1.70 & 2.3678 \\
difference & 0.20 & \\
Unpaired T-Test & $0.16(\mathrm{p}=0.44)$ & \\
Perceived &
\end{tabular}

Perceived Competence

$\begin{array}{lll}\text { Mean } & 5 & 4.12\end{array}$

st.dev $\quad 0.8854 \quad 1.1713$

difference $\quad 0.88$

Unpaired T-Test $\quad 1.42(\mathrm{p}=0,095)$

Results of the IMI are shown. Means and standard deviation of the three subscales have been reported. Furthermore, the difference between means and statistical test for each subscale have also been reported.

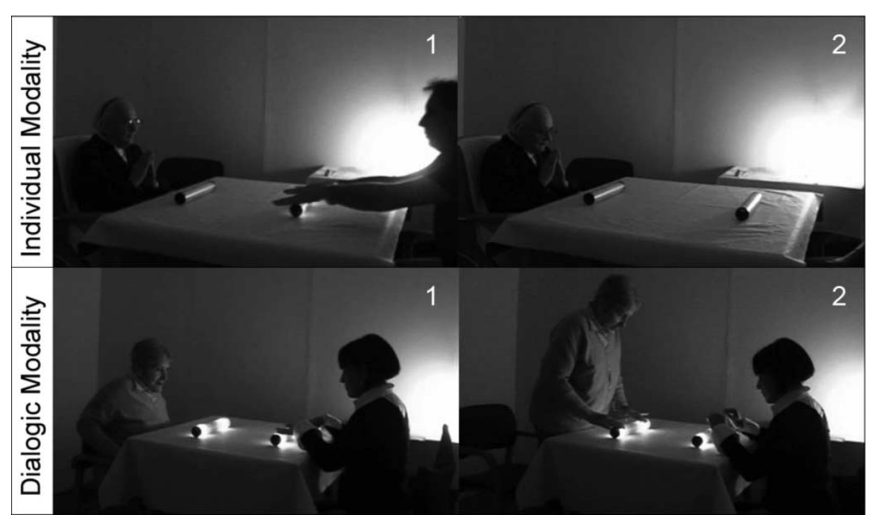

Fig. 17. Two patients during phase A. One of them is working in the individual modality and the other one in the dialogic modality. In 1, both patients observe the therapist while he/she proposes a sensory-motor interaction pattern. In 2 , while the patient in individual condition does not reproduce any interaction patterns, the patient in dialogic modality reproduces the therapist's interaction pattern. It should be noted that this patient expressed the will to stand up in order to better perform the activity.

concerning this scale. However, the interest/enjoyment means difference was significant $\left(t=1.95,{ }^{*} p=0.041\right)$, and this scale was critical in assessing the emergence of the intrinsic motivation [22]. It should be noted that one subject in the individual condition did not agree to answer the questionnaire.

\section{DISCUSSION}

The data derived from the behavioral analysis of phase A show that subjects working in the dialogic condition reproduced a significantly larger number of sensory-motor interaction patterns than subjects working in the individual modality: The dialogic modality favors the spontaneous participation (see Fig. 17) of patients in social exchanges (hypothesis 1).
A post hoc analysis concerning the quality of reproduced patterns in the dialogic condition shows that the subjects on average imitated the therapist's patterns for $78 \%$ (mean $=$ $92.70 \mathrm{~s}$, standard deviation $=59.50$ ) of the time spent using the RP, while for $22 \%$ of the time (mean $=26.70$ s. standard deviation $=45.27$ ), they reproduced a random sensory-motor pattern. These data suggest that the dialogic modality plays a significant role in sustaining the patient in spontaneously initiating the activity and joining the therapist in a dialogic exchange.

Data coming from the behavioral analysis of phase B indicate that in the dialogic condition, the subjects are tuned in with the therapist for a significantly longer time than in the individual condition; furthermore, while in the individual condition the patients performed none, random, and tuning behaviors indifferently without any statistically significant difference among them, in the dialogic condition, they performed the tuning behavior for a significantly longer time than the none and random behaviors. These results clearly indicate that the dialogic condition critically favors the emergence of sensory-motor imitation between the patient and the therapist (hypothesis 2). Therefore, not only does the dialogic condition stimulate the subject to join the activity but also actively sustains the subject in establishing a continuous and solid dialogic exchange.

The data collected from the IMI show that the subjects working in the dialogic condition reported a significantly higher score concerning the interest/enjoyment subscale than subjects working in the individual condition. This subscale constitutes the self-report measurement of the intrinsic motivation: Subjects working in the dialogic condition experience an intrinsic motivation to participate in the activity higher than subjects working in the individual modality (hypothesis 3 ). The appreciable (but not significant) difference between dialogic and individual modalities concerning the positive predictor, i.e., perceived competence, constitutes a further corroboration. Regarding the pressure/tension subscale, there was no difference between the two conditions.

\section{CONCLUSION}

This paper presents a modular robotic system used in the context of dementia care to facilitate nonverbal communication supporting the therapist in maintaining a dialogue with the patient during therapeutic intervention.

Modular robotics offers a remarkable opportunity in the treatment of dementia: The use of simple units, which are easy to manipulate without explicit instruction, puts the subjects at ease and provides them with minimal but clear stimuli to both have a pleasurable experience and perform the tasks that better suit their problem. Furthermore, a dynamic, flexible, and configurable system has proved to be the key factor for obtaining an optimal stimulation tailored to the specific needs of each patient.

The results of the experiment demonstrate the positive effects of the use of the RPs on engagement, coordination, and motivation with regard to therapy in the dialogic condition. In particular, we observed that, differently from the patients working in the individual modality, the patients working in the dialogic modality established with the therapist a nonverbal dialog 
based on sensory-motor imitation of the pattern generated by the therapist.

A number of studies [18], [23] have shown the importance of imitation as a fundamental and universal social function. Imitative behaviors constitute the basic form of communication and are at the basis of early social interactions, thus providing the foundation for future communication. In parent-infant interactions, this reciprocal behavior arises quite naturally and is both spontaneous and unselfconscious [23]. These nonverbal behaviors continue to play a role into adulthood in maintaining social interactions and important conversational activities such as turn taking [24]. Furthermore, a case study conducted by Astell and colleagues on a patient affected by severe dementia illustrates the importance of imitation as a way to keep these types of patients in the social world [18]. Despite a severe cognitive impairment, dementia-affected subjects are still able to engage in social interaction by performing imitative behavior.

In our study, the dialogic component embedded in the RPs plays a positive role in mediating imitative behavior and constitutes the basis of a therapeutic intervention addressed to maintain social interaction.

\section{REFERENCES}

[1] D. N. Ripich, D. Vertes, P. Whitehouse, S. Fulton, and B. Ekelman, "Turntaking and speech act patterns in the discourse of senile dementia of the Alzheimer's type patients," Brain Lang., vol. 40, no. 30, pp. 330-343, 1991.

[2] S. S. Bassuk, T. A. Glass, and L. F. Berkman, "Social disengagement and incident cognitive decline in community," Dwelling Elder. Persons. Ann. Int. Med., vol. 131, no. 3, pp. 165-173, 1999.

[3] D. J. Feil-Seifer and M. J. Mataric, "Defining socially assistive robotics," in Proc. Int. Conf. Rehabil. Robot., Chicago, IL, Jun. 2005, pp. 465-468 (Poster paper).

[4] B. Scassellatti, "How social robots will help us to diagnose, treat and understand autism," presented at the 12th Int. Symp. Robot. Res., San Francisco, CA, Oct. 2005.

[5] B. Robins, K. Dautenhahn, R. Boekhorst, and A. Billard, "Robotic assistants in therapy and education of children with autism: Can a small humanoid robot help encourage social interaction skills?" Universal Access Inf. Soc., vol. 4, no. 2, pp. 105-120, 2005.

[6] P. Marti, M. Bacigalupo, L. Giusti, C. Mennecozzi, and T. Shibata, "Socially assistive robotics in the treatment of behavioural and psychological symptoms of dementia," in Proc. 1st IEEE/RAS-EMBS Int. Conf. Biomed. Robot. Biomechatron., 2006, pp. 483-488.

[7] C. Kidd, W. Taggart, and S. Turkle, "A sociable robot to encourage social interaction among the elderly," in Proc. IEEE Int. Conf. Robot. Autom., Orlando, FL, 2006, pp. 3972-3976.

[8] K. Wada, T. Shibata, T. Saito, and K. Tanie, "Psychological and social effects of robot assisted activity to elderly people who stay at a health service facility for the aged," in Proc. IEEE Int. Conf. Robot. Autom., Sep. 2003, pp. 3996-4001.

[9] P. Marti, H. H. Lund, M. Bacigalupo, L. Giusti, and C. Mennecozzi, "Blending senses: A multi-sensory environment for the treatment of dementia affected subjects," J. Gerontechnol., vol. 6, no. 1, pp. 33-41, 2006.

[10] P. Marti and L. Giusti, "Coupling the digital and the physical in therapeutic environments," in Proc. 12th Int. Conf., HCI Int., Part II, Hum.-Comput. Interaction. Interaction Platforms Techn. (Lecture Notes in Computer Science 4551). Berlin, Germany: Springer-Verlag, Jul. 22-27, pp. 11731182.

[11] H. H. Lund and P. Marti, "Designing modular robotic playware," to be presented at the IEEE Int. Workshop Robots Human Interactive Commun., Toyama, Japan, Sep. 27-Oct. 2

[12] H. H. Lund and P. Marti, "Designing manipulative technologies for children with different abilities," Artif. Life Robot., vol. 9, no. 4, pp. 175-187, 2005.

[13] J. Nielsen and H. H. Lund, "Modular robotics as a tool for education and entertainment," Comput. Human Behav., vol. 24, no. 2, pp. 234-248, 2008.
[14] H. H. Lund, Robots at Play. Odense, Denmark: Southern Denmark Univ., 2007, 160 pp.

[15] R. A. Brooks, "A robust layered control system for a mobile robot," IEEE J. Robot. Autom., vol. 2, no. 1, pp. 14-23, Mar. 1986.

[16] H. H. Lund, M. D. Pedersen, and R. Beck, "Modular robotic tiles experiments for children with autism," Artif. Life Robot., vol. 13, no. 2, pp. 394-400, 2009.

[17] D. Aldridge. (2001). Music therapy and neurological rehabilitation Recognition and the performed body in an ecological niche. Music Therapy Today [Online]. Available: www.musictherapyworld.info

[18] A. J. Astell and M. Ellis, "The social function of imitation in severe dementia," Infant Child Dev., vol. 15, pp. 311-319, 2006.

[19] H. H. Lund, P. Marti, A. Derakhshan, R. Beck, T. Kiltbo, and J. Nielsen, "Modular robotics for novel tools in dementia treatment," in Proc. RoMan IEEE Int. Workshop Robots Human Interactive Commun., 2006, pp. 655-661.

[20] J. Itten, The Art of Color: The Subjective Experience and Objective Rationale of Color. New York: Van Nostrand Reinhold, 1973.

[21] M. F. Folstein, S. E. Folstein, and P. R. McHugh, "Mini-mental state: A practical method for grading the state of patients for the clinician," $J$ Psychiatr. Res., vol. 12, pp. 189-198, 1975.

[22] R. W. Plant and R. M. Ryan, "Intrinsic motivation and the effects of selfconsciousness, self-awareness, and ego-involvement: An investigation of internally-controlling styles," J. Pers., vol. 53, pp. 435-449, 1985.

[23] M. Tomasello, "The social bases of language acquisition," Soc. Dev., vol. 1, no. 1, pp. 67-87, 1992.

[24] H. Sacks, E. A. Schegloff, and G. Jefferson, "A simplest systematics for the organization of turn-taking for conversation," Language, vol. 50, pp. 696-735, 1974.

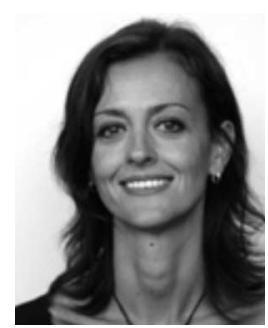

Patrizia Marti is currently an Assistant Professor of human computer interaction and design of learning technologies at the University of Siena, Siena, Italy, where she coordinates a research group in interaction design with the Department of Communication Science. She has a long-standing track record of research in interaction design and the ethnographic study of social practice to inform the design of information systems, in participatory design, and evaluation with end users. She has been a Principal Researcher for a number of European and national funded projects in the areas of human-robot interaction, health care, and ubiquitous computing.

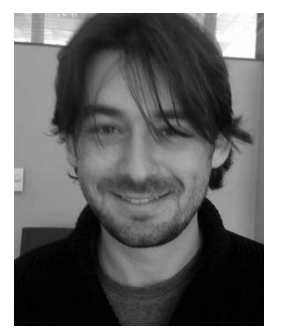

Leonardo Giusti received the Ph.D. degree in telematics and information society from the University of Florence, Florence, Italy, in 2008.

$\mathrm{He}$ is currently a Researcher with the Fondazione Bruno Kessler (FBK-irst), Povo, Italy. He is also with the Department of Communication Science, University of Siena, Siena, Italy, where he teaches cognitive psychology and human-machine interface. Since 2003, he has been an Interaction Designer for a number of national and European projects in the area of robotic technologies for playful, educational, and therapeutic activities. He has authored or coauthored several papers in conference proceedings and journals in the area of human-computer interaction and human-robot interaction. $\mathrm{He}$ also teaches graphic design at the University of Trento, Trento, Italy.

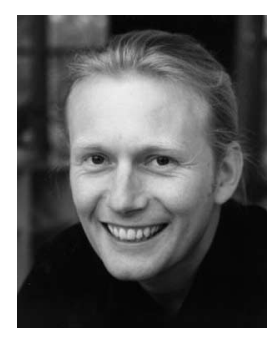

Henrik Hautop Lund is currently a Full Professor and the Head of the Center for Playware, Technical University of Denmark, Kongens Lyngby, Denmark, where he is also the Director of Entertainment Robotics. He recently founded the Center for Playware to focus even further on how playful aspects of robotics may provide motivation for any citizen to perform different kinds of interaction with the robots of our future daily life. He has authored or coauthored more than 125 scientific articles in the field of robotics. He founded and headed the LEGO Laboratory in 1997-2000 and invented the RoboCup Junior robot football game for children. He also founded RoboCluster.

Prof. Lund has been a member of the Danish Research Council. His Adaptronics Group won the 2002 RoboCup Humanoids Free Style World Championship. 